\title{
Differences in COVID-19-Related Testing and Healthcare Utilization by Race and Ethnicity in the Veterans Health Administration
}

\author{
Javad Razjouyan ${ }^{1,2,3} \cdot$ Drew A. Helmer ${ }^{1,2} \cdot$ Ang Li $^{1,2} \cdot$ Aanand D. Naik ${ }^{1,2} \cdot$ Christopher I. Amos ${ }^{1,2} \cdot$ Venkata Bandi $^{1,2}$. \\ Amir Sharafkhaneh ${ }^{1,2}$
}

Received: 18 October 2020 / Revised: 25 January 2021 / Accepted: 27 January 2021 / Published online: 10 March 2021

(C) This is a U.S. government work and not under copyright protection in the U.S.; foreign copyright protection may apply 2021

\begin{abstract}
Importance Recent reports indicate differences in COVID-19-related care and outcomes between Black and White Americans. Objective We examine the COVID-19-related healthcare utilization and mortality by race and ethnicity of patients tested for SARS-CoV-2 in the Veterans Health Administration (VHA).

Design A retrospective cohort study.

Setting We used the VHA COVID-19 shared data resources between February 1 and June 30, 2020.

Participants Veterans tested for SARS-CoV-2 virus by VHA.

Exposure(s) Three racial-ethnicity groups of Black, Hispanic, and White (as reference) veterans.

Main Outcome(s) and Measure(s) Main outcomes are testing rate, positivity rate, hospitalization rate, ICU admission rate, and inhospital mortality. Controlling for sex, age, and Elixhauser comorbidity index, we report adjusted odds ratios (aOR) and 95\% confidence intervals $(95 \% \mathrm{CI})$ from logistic regression models.

Results Of the 8,667,996 active veteran enrollees, 252,702 were tested by VHA from February to June, 2020, with 20,500 positive results and 4,790 hospitalizations. The testing rate was $4.4 \%$ among Black and $4.7 \%$ among Hispanic veterans compared to White veterans, $2.8 \%$. The testing positivity rate was similarly elevated among Black (12.2\%) and Hispanic (11.6\%) veterans compared to White veterans $(6.0 \%)$. The aORs of hospitalization in Black veterans $(1.88 ; 95 \%$ CI 1.74, 2.03) and Hispanic
\end{abstract}

Key Points Question: Do data from the Veterans Health Administration (VHA), an integrated, mission-focused healthcare system with national scope, demonstrate racial and ethnic disparities in COVID-19-related healthcare and outcomes?

Findings: Between February and June 2020, Black and Hispanic veterans had higher SARS-CoV-2 testing and test positivity rates compared to White veterans. Among those hospitalized, ICU transfer and in-hospital mortality rates were similar.

Meaning: Over the initial part of the SARS-CoV-2 epidemic in the U.S., VHA provided more initial COVID-19-related care to Black and Hispanic veterans compared to White veterans, likely reflecting social determinants of health, factors affecting access to non-VHA care, or a preference for VHA care in these groups.

\author{
Amir Sharafkhaneh \\ amirs@bcm.edu; SHARAFKHANEH.AMIR@va.gov \\ Javad Razjouyan \\ javad.razjouyan@bcm.edu; javad.razjouyan@va.gov \\ Drew A. Helmer \\ Drew.Helmer@bcm.edu; Drew.Helmer@va.gov \\ Ang Li \\ Ang.Li2@bcm.edu \\ Aanand D. Naik \\ anaik@bcm.edu; Aanand.Naik@va.gov
}

\section{Christopher I. Amos \\ Chris.Amos@bcm.edu \\ Venkata Bandi \\ Venkata.Bandi@va.gov}

1 VA HSR\&D Center for Innovations in Quality, Effectiveness and Safety, Michael E. DeBakey VA Medical Center, Houston, TX 77030, USA

2 Department of Medicine, Baylor College of Medicine, Michael E. DeBakey VA Medical Center, Houston, TX 77030, USA

3 Big Data Scientist Training Enhancement Program (BD-STEP), VA Office of Research and Development, Washington, DC, USA 
veterans $(1.41 ; 95 \%$ CI $1.25,1.60)$ were higher compared to White veterans. No significant differences by race and ethnicity were observed in OR or aOR of ICU admission and in-hospital death among hospitalized patients.

Conclusions and Relevance On a national level, the VHA was more likely to test and hospitalize Black and Hispanic veterans compared to White veterans, but there were no significant differences in ICU admission or in-hospital mortality among those hospitalized. This pattern of differences may relate to social determinants of health, factors affecting access to non-VHA care, or preferences for VHA care affecting initial care seeking, but not in-hospital outcomes.

Keywords COVID19 $\cdot$ Disparity $\cdot$ Prevalence $\cdot$ Veteran

\section{Background}

Initial experience suggests that Black and Hispanic Americans suffer and die from COVID-19 at disproportionately higher rates than white Americans [1]. A county-level analysis indicated that disproportionately Black counties had more COVID-19 diagnoses and deaths than all other counties [2]. National surveillance data suggests that Black and Hispanic Americans experienced higher COVID-19 case and hospitalization rates from January 22 to May 30, 2020, although race and ethnicity information was missing for half of the sample [3]. Similar patterns were observed in New York [4] and early reports from US Department of Veterans Affairs (VHA) [5]. Studies from Georgia also showed higher hospitalization and higher mechanical ventilation rates among Blacks compared to Whites [6,7]. In contrast, several studies reported no association between race and in-hospital mortality [4-8]. Limitations of these studies include limited size and geographic representation of the samples, missing information on race and ethnicity, imbalanced representation of race and ethnicity within the samples, and early data collection termination. There is an urgency to better understand the associations between race and ethnicity and COVID-19-related care and outcomes (Fig. 1).

VHA is a national integrated healthcare provider which operates more than 150 medical centers and more than 1,000 outpatient clinics across the USA. It provides medical care and related social services for more than nine million enrolled veterans with robust racial and ethnic diversity generally representative of the military population [9]. The VHA has consistently been shown to provide care with little evidence of racial and ethnic disparities [10-12]. This is commonly attributed to a veteran-focused mission, eligibility criteria based primarily on military service and income, low out-of-pocket cost of care, and provision of care in diverse communities across the nation [13].

The objective of this report is to compare by race and ethnicity the pattern of positive COVID-19 testing, hospitalization and intensive care unit (ICU) admission, and in-hospital mortality among veterans who utilize the VHA for SARS$\mathrm{CoV}-2$ testing. We hypothesized that these parameters are not different among race and ethnic groups.

\section{Methods}

\section{Study database, design, and population}

We performed a retrospective cohort study using the VHA's Corporate Data Warehouse (CDW), which is a relational database that aggregates patient data from all VHA facilities from 1999 to the present [14]. We used the COVID-19 Shared Data Resource that contains information related to COVID-19 treated within VHA. It encompasses a wide range of information of SARS-CoV-2 tested patients (e.g., timing and nature of test results, pharmacological and nonpharmacological interventions, patient outcomes, and preexisting conditions and medication).

For the present study, we included all veterans who were active users of VHA services and excluded patients who did not have at least one outpatient visit or one inpatient stay in calendar years 2018 and 2019. From this population, we identified all VHA veteran patients who underwent SARS-CoV-2 testing at a VHA facility or outside lab between February 1 and June 30, 2020. A patient was considered a case if he/she had a positive reverse transcription polymerase chain reaction (RT PCR) SARS-CoV-2 test. We then defined COVID-19related hospitalization to be the incident admission that occurred within 7 days after the SARS-CoV-2 index date. The index date was defined as the date of the first positive SARSCoV-2 test or the hospitalization admission date within 15 days before the positive test date. Patients were followed for a minimum of 30 days unless death or hospital discharge occurred (dataset locked on July 30, 2020). The study was approved by the institutional review board (IRB) of Baylor College of Medicine and the Research and Development Committee of the Michael E. DeBakey VA Medical Center.

\section{Key study Variables}

Our key variable included a combination of race and ethnicity using VHA facility-reported data [15]. Non-Hispanic White (White), Non-Hispanic Black (Black), and Hispanic veterans (the labels used in data collection at enrollment) formed the key comparison groups. Other race categories, including 


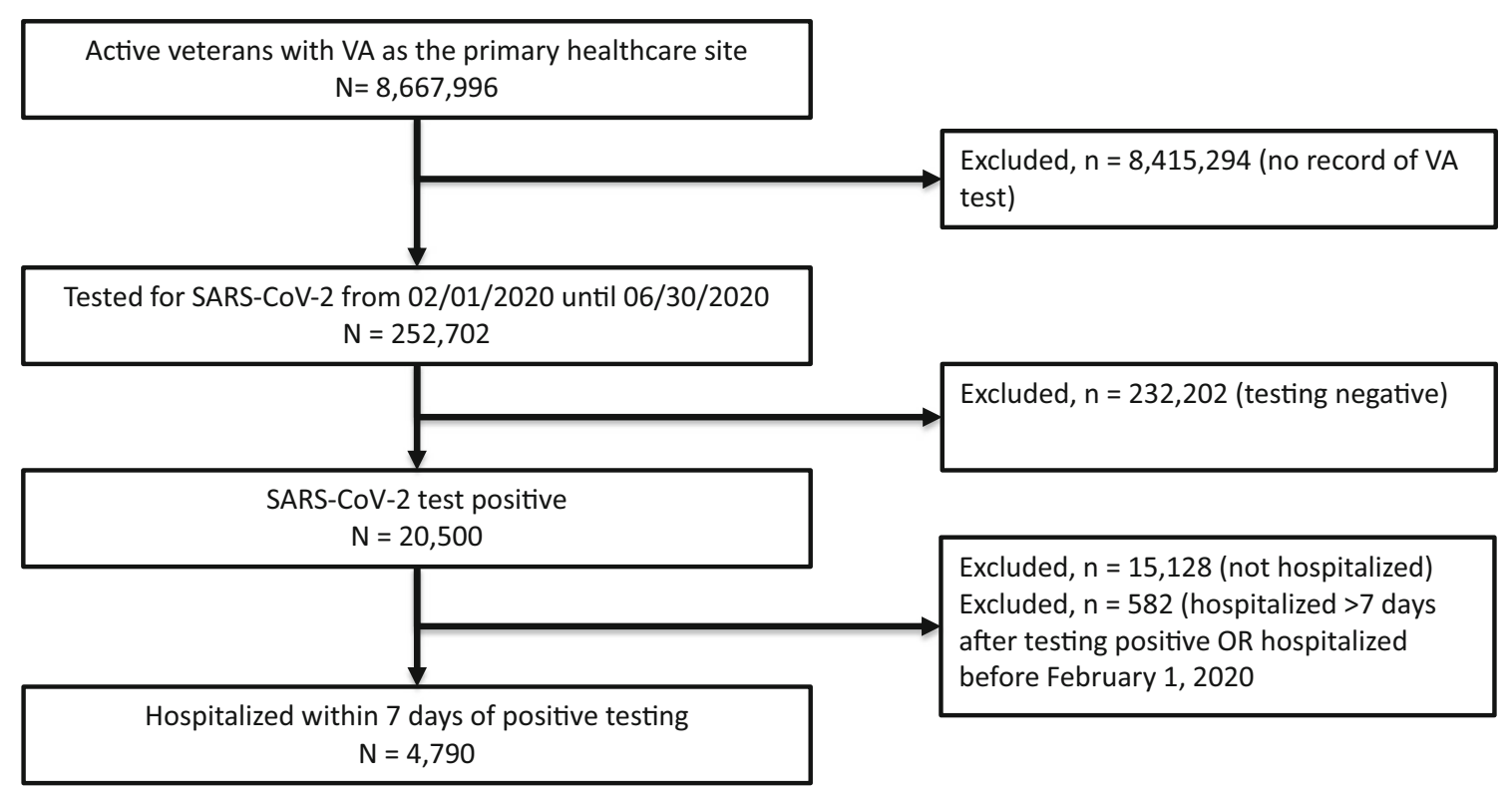

Fig. 1 CONSORT diagram of patients tested for SARS-CoV-2 in VHA facilities and hospitalized due to COVID-19 infection

Asian, American Indian, and Unknown, are reported, but not included in statistical modeling due to low sample size.

Our primary outcomes of the statistical modeling included in-hospital mortality and ICU admission. Mortality was assessed from the patient treatment file [16]. ICU admission was assessed using inpatient transfer-to-specialty data fields [17]. In addition, we report the testing rate and positivity rate overall and for each race and ethnicity category. Testing rate is the number tested for SARS-CoV-2 during the study period divided by the total enrolled in VHA care. Positivity rate is the number of positive SARS-CoV-2 tests divided by the number tested.

Potential confounders of COVID-19 disease severity were abstracted from the VA COVID-19 Shared Data Resources [18]. These included age, sex, Elixhauser comorbidity index $[19,20]$, and selected underlying medical conditions associated with the most consistently increased risk for severe illness as reported by the Centers for Disease Control and Prevention (CDC) as of July 17, 2020: cancer; chronic kidney disease; chronic obstructive pulmonary disease; obesity with body mass index $(\mathrm{BMI}) \geq 30$; serious heart problems, including heart failure, coronary artery disease, and cardiomyopathy; sickle cell disease; and type 2 diabetes [9]. Elixhauser index score and individual comorbid condition were determined using International Classification of Diseases (ICD)-10 codes from the 2 years prior to index date.

\section{Statistical Analysis}

To characterize the cohort, continuous variables are presented as mean and standard deviation, and categorical variables as number and percentage. We used logistic regression models to test the association between race and ethnicity (with non-
Hispanic White as the reference group) and each outcome of interest (i.e., testing rate, positivity rate, mortality, and ICU admission). Odds ratios (OR) with $95 \%$ confidence intervals ( $95 \% \mathrm{CI}$ ) were reported. Each model was adjusted by age, sex, and Elixhauser comorbidity index. We performed a sensitivity analysis adjusting the models for all outcomes using individual key comorbidities instead of the Elixhauser Comorbidity index. Subgroup analysis of in-hospital mortality was performed within the ICU admitted patients in addition to the entire hospitalized group. All statistical analyses were performed using SPSS, version 26 (SPSS Inc, Chicago, Illinois).

\section{Results}

\section{Prevalence of SARS-CoV-2 testing and positivity among different racial and ethnic groups}

Among the 8,667,996 active veterans, 252,702 were tested for SARS-CoV-2 from February to June 30, 2020. Among those tested, 151,836 (60.1\%) were White, 60,529 (24.0\%) were Black, 20,612 (8.2\%) were Hispanic, 2,549 (1.0\%) were Asian, 1,795 (0.7\%) were American Indian or Alaska Native, and 15,381 (6.1\%) unknown. The overall testing rate was $2.9 \%$ (Table 1, supplement Table 1); Blacks (4.4\%; OR = 1.60, 95\% CI 1.58-1.61) and Hispanics (4.7\%; OR = 1.70, 95\% CI 1.68-1.73) had higher testing rates compared to Whites (2.8\%). Among the tested veterans, 20,500 were positive for an overall positivity rate of $8.1 \%$. Among those positive, 9,162 (44.7\%) were White, 7398 (36.1\%) were Black, 2,400 (11.7\%) were Hispanic, 175 (0.9\%) were Asian, 159 $(0.8 \%)$ were American Indian or Alaska Native, and 1,206 (5.9\%) unknown. The positivity proportion among Blacks 
Table 1 COVID-19 testing among different races and ethnicities of veterans who used VA healthcare services in the past 2 years

\begin{tabular}{lllllll}
\hline & White & Black & Hispanic & Asian & American Indian or Alaska native & Unknown \\
\hline Total, $N$ & $5,408,807$ & $1,372,986$ & 439,725 & 99,597 & 79,433 & $1,267,448$ \\
Tested, $N(\%$ tested/total $)$ & $151,836(2.8)$ & $60,529(4.4)$ & $20,612(4.7)$ & $2,549(2.6)$ & $1,795(2.3)$ & $15,381(1.2)$ \\
Positive, $N(\%$ positive/tested $)$ & $9,162(6.0)$ & $7,398(12.2)$ & $2,400(11.6)$ & $175(6.9)$ & $159(8.9)$ & $1,206(7.8)$ \\
Hospitalized, $N$ & $1,915(20.9)$ & $2,138(28.9)$ & $432(18.0)$ & $39(22.3)$ & $31(19.5)$ & $235(19.5)$ \\
$(\%$ hospitalized/positive $)$ & & & & & & \\
\hline
\end{tabular}

$(12.2 \% ; \mathrm{OR}=2.17,95 \% \mathrm{CI} 2.10-2.24)$ and Hispanics $(11.6 \%$; OR $=2.05,95 \%$ CI 1.96-2.15) were also higher compared to Whites $(6.0 \%)$.

\section{Incidence of COVID-19 severe illness among different racial and ethnic groups}

A total of 4,790 veterans (23.4\% of those tested) had an incident hospitalization in VHA attributable to COVID-19 infection (Table 2). The hospitalization rate was highest among Blacks $(n=28.9 \%)$, then Whites $(n=20.9 \%)$, then Hispanics $(n=18.0 \%)$. The number of hospitalizations among other race and ethnicity categories was much lower. The average length of stay (LoS) in Whites was higher (12.2 days) compared to Blacks (11.3 days) and Hispanics (10.3 days). Among those hospitalized, Whites (71.8 years) were older than Blacks (66.9 years) and Hispanics (63.0 years). The BMI (mean $30 \mathrm{~kg} / \mathrm{m}^{2}$ ) and sex distribution (95\% male) were similar among the White, Black and Hispanic patients. The average Elixhauser comorbidity index in White patients (14.2) was higher than Black (13.8) and Hispanic (9.2) veterans. Key comorbidity distribution differed significantly among the different race and ethnic groups, with the highest prevalence of each comorbidity in White and the lowest in Hispanic patients with the exception of cancer and chronic kidney disease (Table 2).

The statistical comparison of COVID-19 hospitalization, ICU admission, and mortality by Black, White, and Hispanic race and ethnicity categories is summarized in Table 3. The unadjusted OR of hospitalization was 1.54 (95\% CI 1.43-1.65) in Blacks vs. Whites and 0.83 (95\% CI 0.74-0.93) in Hispanics vs. Whites. After adjusting for age, sex, and Elixhauser comorbidity index, the adjusted OR of hospitalization was appreciably higher at $1.88(95 \% \mathrm{CI}$ 1.74-2.03) for Blacks vs. Whites and 1.41 (95\% CI 1.251.60) for Hispanics vs. Whites.

Table 2 Characteristics of 4,790 veterans hospitalized with SARS-CoV-2 positive tests

\begin{tabular}{|c|c|c|c|c|c|c|}
\hline & White & Black & Hispanic & $\begin{array}{l}\text { American Indian or } \\
\text { Alaska native }\end{array}$ & Asian & Unknown \\
\hline$N(\%)$ & 1,915 & 2,138 & 432 & 39 & 31 & 235 \\
\hline Age [years], mean (SD) & $71.8(13.4)$ & $66.9(12.5)$ & $63.0(16.5)$ & $60.7(20.3)$ & $66.3(14.5)$ & $67.8(16.1)$ \\
\hline BMI [Kg/m^2], mean (SD) & $29.4(7.3)$ & $29.5(7.2)$ & $30.2(6.8)$ & $27.7(5.8)$ & $29.9(6.3)$ & $28.2(6.4)$ \\
\hline Gender [number Male], $N(\%)$ & $1829(96)$ & $2015(94)$ & $412(95)$ & $36(92)$ & $29(94)$ & $223(95)$ \\
\hline Length of stay [days], mean (SD) & $12.1(11.9)$ & $11.3(11.5)$ & $10.3(10.6)$ & $10.1(7.8)$ & $11.9(11.1)$ & $11.2(10.6)$ \\
\hline ICU admission, $n(\%)$ & $795(41.5)$ & $919(43.0)$ & $181(41.9)$ & $18(46.2)$ & $15(48.4)$ & $87(37.0)$ \\
\hline In-hospital mortality, $n(\%)$ & $337(17.6)$ & $331(15.5)$ & $64(14.8)$ & $6(15.4)$ & $7(22.6)$ & $33(14.0)$ \\
\hline \multicolumn{7}{|l|}{ Comorbidities and preexisting conditions* } \\
\hline Elixhauser comorbidity index, mean (SD) & $13.4(14.2)$ & $12.8(13.8)$ & $9.2(12.0)$ & $6.1(9.0)$ & $13.0(12.6)$ & $9.6(12.9)$ \\
\hline Congestive heart failure, $N(\%)$ & $371(19)$ & $366(17)$ & $61(14)$ & $3(8)$ & $5(16)$ & $26(11)$ \\
\hline Coronary atherosclerosis and other heart disease, $N(\%)$ & $673(35)$ & $545(25)$ & $120(28)$ & $11(28)$ & $9(29)$ & $52(22)$ \\
\hline Cardiomyopathy, $N(\%)$ & $134(7)$ & $175(8)$ & $26(6)$ & $4(10)$ & $1(3)$ & $12(5)$ \\
\hline Cancer, $N(\%)$ & $513(27)$ & $652(30)$ & $90(21)$ & $9(23)$ & $5(16)$ & $44(19)$ \\
\hline Chronic kidney disease, $N(\%)$ & $498(26)$ & $700(33)$ & $80(19)$ & $6(15)$ & $7(23)$ & $56(24)$ \\
\hline Chronic obstructive pulmonary disease, $N(\%)$ & $596(31)$ & $489(23)$ & $68(16)$ & $6(15)$ & $10(32)$ & $37(16)$ \\
\hline Obesity (BMI $\geq 30), N(\%)$ & $149(8)$ & $175(8)$ & $40(9)$ & $2(5)$ & $2(6)$ & $8(3)$ \\
\hline Sickle cell disease, $N(\%)$ & $1(0)$ & $14(1)$ & $1(0)$ & $0(0)$ & $0(0)$ & $0(0)$ \\
\hline Diabetes type $2, N(\%)$ & $881(46)$ & $1115(52)$ & $200(46)$ & $16(41)$ & $23(74)$ & $82(35)$ \\
\hline
\end{tabular}

$B M I$ body mass index, *Comorbidities and preexisting conditions estimated for 2 years prior to positive test date 
Table 3 Association between race and ethnicity and COVID-19 hospitalization, ICU admission, and mortality among Black, White, and Hispanic veterans

Black vs. white

Odds ratio $(95 \% \mathrm{CI})$
Adjusted odds ratio $(95 \% \mathrm{CI})$

\section{Hispanic vs. white}

Odds ratio $(95 \% \mathrm{CI})$

Adjusted odds ratio $(95 \% \mathrm{CI})$

\begin{tabular}{|c|c|c|c|c|}
\hline \multicolumn{5}{|l|}{ Among all positive cases $(n=20,500)$} \\
\hline Hospitalized for SARS-CoV-2, Unadjusted & $1.54(1.43,1.65)$ & $1.88(1.74,2.03)$ & $0.83(0.74,0.93)$ & $1.41(1.25,1.60)$ \\
\hline \multicolumn{5}{|l|}{ Among hospitalized patients $(n=4,790)$} \\
\hline ICU admission, unadjusted & $1.06(0.94,1.20)$ & $1.08(0.95,1.23)$ & $1.02(0.82,1.26)$ & $1.07(0.86,1.33)$ \\
\hline In-hospital death, unadjusted & $0.86(0.73,1.01)$ & $1.16(0.97,1.38)$ & $0.81(0.61,1.09)$ & $1.22(0.90,1.66)$ \\
\hline \multicolumn{5}{|l|}{ Among ICU-admitted patients $(n=2,015)$} \\
\hline In-hospital death, unadjusted & $0.81(0.69,0.96)$ & $1.11(0.93,1.33)$ & $0.82(0.61,1.11)$ & $1.22(0.89,1.67)$ \\
\hline
\end{tabular}

*Adjusted by age, sex, Elixhauser comorbidity index (includes obesity)

The unadjusted OR of ICU admission was 1.06 (95\% CI $0.94,1.20)$ in Blacks vs. Whites and 1.02 (95\% CI 0.82, 1.26) in Hispanics vs. Whites. After adjusting for age, sex, and Elixhauser comorbidity index, the adjusted OR of ICU admission was 1.08 (95\% CI 0.95, 1.23) for Blacks vs. Whites and 1.07 (95\% CI 0.86, 1.33) for Hispanics vs. Whites.

The overall in-hospital mortality occurred in 778 hospitalized patients $(16.2 \%)$ with a median time to death of 11 days from the index date. Frequency of death among white and black hospitalized patients was comparable (Whites $n=337,43.3 \%$; Blacks $n$ $=331,42.5 \%)$ and higher than Hispanic patients $(n=64,8.2 \%)$. However, the unadjusted OR of in-hospital mortality was not different among the groups $[0.86(95 \%$ CI $0.73,1.01)$ in Blacks vs. Whites and 0.81 (95\% CI 0.61, 1.09) in Hispanics vs. Whites]. After adjusting for age, sex, and Elixhauser comorbidity index, the adjusted OR of in-hospital mortality remained comparable among the groups $[1.16(95 \%$ CI $0.97,1.38)$ for Blacks vs. Whites and 1.22 (95\% CI 0.90, 1.66) for Hispanics vs. Whites]. In the ICU subgroup analysis, the odds of mortality were similarly comparable across groups [aOR 1.11 (95\% CI $0.93,1.33)$ for Blacks vs. Whites and aOR 1.22 (95\% CI 0.89, 1.67) for Hispanics vs. Whites].

Our sensitivity analysis, replacing the Elixhauser with the individual comorbid conditions (i.e., cancer; chronic kidney disease; chronic obstructive pulmonary disease; obesity with body mass index $(\mathrm{BMI}) \geq 30$; serious heart problems, including heart failure, coronary artery disease, and cardiomyopathy; sickle cell disease; and type 2 diabetes), did not substantially alter our findings (Supplemental Table 1).

\section{Discussion}

In this analysis of a large, national, retrospective cohort of veterans cared for by the VHA health care system, we found that Black and Hispanic veterans were more likely to be tested for SARS-CoV-2, more likely to test positive for SARS-CoV2 , and more likely to be hospitalized in VHA facility compared to White veterans. However, among hospitalized patients, these racial and ethnic groups had similar odds of ICU admission and in-hospital mortality. Our results support the disproportionate risk of contracting COVID-19 among Black and Hispanic patients, likely due to social determinants of health, but indicate that equitable access to testing and treatment may mitigate severe adverse outcomes attributable to COVID-19 among hospitalized patients.

After controlling for demographics (i.e., age and sex) and comorbidities, we did not observe an appreciable difference in in-hospital mortality and ICU admissions among racial and ethnic groups in this national cohort. Our studies complement other recent publications but provide a more complete analysis of key aspects of healthcare utilization in a national sample with more complete data. Two studies from Georgia showed that Blacks treated in a private sector healthcare system were more likely to be hospitalized than non-Blacks, while invasive mechanical ventilation or in-hospital mortality between Black and non-Black patient population are the same [6,7]. Both of these studies had smaller samples, over-representation of Black patients, categorized patients as Black or non-Black (disregarding other race and ethnicity details), and were focused on the same metropolitan area. Our findings are also consistent with a study in Louisiana with 1,382 hospitalized cases. The authors reported that Black race was not associated with higher in-hospital mortality than White race, after adjusting with confounding factors [8]. Limitations of this study included a single, private healthcare provider in a limited geography, and an exclusive focus on Black and White patients. A VHA national study also showed no disparity in in-hospital mortality among minorities compared to Whites. However, similar to our findings, they observed a disparity between race and ethnicity in testing positive. They reported the VHA cohort only until May 4, 2020, and did not consider 
the VHA hospitalization rate. A separate study from New York City showed a non-significant increased hazard of inhospital mortality among Black when compared with Whites. The authors reported a higher likelihood of hospitalization among Blacks compared to Whites while Hispanics were more likely to experience in-hospital mortality compared to Whites. This study was limited to a single medical center at the epicenter of COVID-19 disease and confined to a short time interval (March 10, 2020, to April 13, 2020) [4].

The lack of racial and ethnic disparities in the clinical outcomes among veterans hospitalized with COVID-19 in VHA facilities is consistent with the literature examining racial and ethnic disparities in VHA health care, more generally. Studies of VHA care consistently find lower likelihood of racial and ethnic disparities than those reported in the private sector [10-12]. By statute and policy, VA healthcare provides equivalent access to health care and reduces out-of-pocket expenses for all eligible veterans. Because of its mission to serve all eligible veterans, VHA may, therefore, partially reduce bias that contributes to racial disparities observed in other health care settings [13].

The higher rates of SARS-CoV-2 testing positivity and hospitalization among Black and Hispanic veterans suggest the critical importance of factors prior to SARS-CoV-2 testing in these groups. Salient factors contributing to the higher observed rates among Black and Hispanic veterans may include greater exposure to SARS-CoV-2 through employment, housing, or other social factors [21], higher susceptibility to infection [22, 23], delays in health care and hospitalization, and/or intrinsic higher risk of mortality once infected [24]. Social determinants of health and implicit bias have measurable adverse effects on access to healthcare and quality of care among Black and Hispanic Americans [25]. Although the VHA data do not contain relevant information, our results are consistent with evidence supporting the importance of social determinants of health as major contributors to racial and ethnic disparities in health outcomes. Supplementing VHA data with additional information about social determinants of health would facilitate our understanding of these relationships and could lead to new approaches to improving public health.

Why are Black and Hispanic veterans more likely than White veterans to seek COVID-19-related care at the VHA? It is plausible, although we could not analyze this directly, that barriers to care in the private sector, including a lack of healthcare insurance coverage, perceptions of high cost or low quality at available non-VHA providers, and perceived racial and ethnic bias may have contributed to the relatively higher use of COVID-19-related care at VHA facilities by Black and Hispanic veterans. There is evidence suggesting that some veterans prefer to use VHA services over private sector alternatives even when given the choice and that this preference may be more prevalent among Black and Hispanic veterans $[26-30]$.

\section{Strengths and Weaknesses}

The strengths of the study include a large cohort of patients from a healthcare system of national scope with a distribution of race and ethnicity approximating the national population. Furthermore, we used a well-curated dataset with near complete race and ethnicity data and up-to-date information of direct clinical and operational relevance to accurately assess for testing, results, and outcomes. Although we applied a standard cross-sectional analytic approach to examine the association between race and ethnicity and health outcomes, a more sophisticated analysis at the local level addressing geographic spread of infection over time could reveal a more nuanced understanding. The limitations of the study also include potential missing information from veterans hospitalized in nonVHA facilities who were included in our analysis based on a positive test for SARS-CoV-2 performed by VHA. To the extent non-VHA hospitalizations occurred more frequently among White veterans, the unobservable total hospitalization rate (VHA and non-VHA) might have erased the differences observed by race and ethnicity in our data. The mortality assessment was limited to hospitalized patients only due to delays in reporting out-of-hospital deaths. Our data did not capture all possible confounding factors, including the social determinants of health that may explain the differences seen among race and ethnicity groups. We were also unable to capture objective measures of the severity of COVID-19 at the time of admission or the severity of the comorbid conditions. A shortcoming of the Elixhauser comorbidity index is imperfect capture of burden of disease and loss of information about individual comorbidities. However, our sensitivity analysis including each major comorbid condition in the model instead of the Elixhauser comorbidity index did not substantively change our findings.

\section{Conclusion}

Our analyses of this large, national cohort of VHA active users showed that testing and test positivity rates of SARS-CoV-2 were significantly higher in Black and Hispanic veterans relative to White veterans, as were VHA hospitalizations, but the clinical outcomes of ICU admission and in-hospital mortality rates were similar among the hospitalized subset. These findings reinforce the importance of pre-hospitalization factors, including social determinants of health and barriers to private sector care, in the risk of contracting COVID-19 and accessing first-line care.

Supplementary Information The online version contains supplementary material available at https://doi.org/10.1007/s40615-021-00982-0. 
Funding The study is funded by seed funding from Baylor College of Medicine, Houston, Texas, USA, and the Center for Innovations in Quality, Effectiveness and Safety (VA HSRD CIN 13-413), Michael E. DeBakey VA Medical Center, Houston, Texas, USA. The views expressed in this article are those of the authors and do not necessarily reflect the position or policy of the Department of Veterans Affairs or the United States government.

\section{Declarations}

Ethics Approval The study was approved by the institutional review board (IRB, IRB\#: H-47595) of Baylor College of Medicine and the Research and Development Committee of the Michael E. DeBakey VA Medical Center. All procedures performed in studies involving human participants were in accordance with the ethical standards of the institutional and/or national research committee and with the 1964 Helsinki declaration and its later amendments or comparable ethical standards.

Informed Consent This is a secondary database analysis on a national large cohort of patients. In line with Baylor College of Medicine and VHA policies for large national database, we requested a waiver of consent and HIPAA authorization. This type of study would not be possible without a waiver of individual patient consent to access PHI. In addition, due to the nature of the virus, it would be difficult to contact all of these patients as some of them may have died. Furthermore, in person recruitment is currently prohibited due to social distancing orders that have been put in place. All data analyzed in the VA Informatics and Computing Infrastructure (VINCI) behind VA firewall. VINCI provides researcher access to VA data and to facilitate the analysis of those data while ensuring veterans' privacy and data security.

Conflict of Interest The authors declare no competing interests.

Disclaimer The views expressed in this article are those of the authors and do not necessarily reflect the position or policy of the Department of Veterans Affairs or the United States government.

\section{References}

1. Evans MK. Covid's Color Line - Infectious Disease, Inequity, and Racial Justice. N Engl J Med. 2020;383(5):408-10.

2. Millett GA, Jones AT, Benkeser D, Baral S, Mercer L, Beyrer C, et al. Assessing differential impacts of COVID-19 on black communities. Ann Epidemiol. 2020;47:37-44.

3. Stokes EK, Zambrano LD, Anderson KN, Marder EP, Raz KM, el Burai Felix S, et al. Coronavirus Disease 2019 Case Surveillance United States, January 22-May 30, 2020. MMWR Morb Mortal Wkly Rep. 2020;69(24):759-65.

4. Renelus BD, et al., Racial disparities in COVID-19 hospitalization and in-hospital mortality at the height of the New York City pandemic. J Racial Ethn Health Disparities. 2020;1-7.

5. Rentsch CT, et al. Covid-19 by race and ethnicity: a national cohort study of 6 million United States veterans. medRxiv. 2020.

6. Killerby ME, Link-Gelles R, Haight SC, Schrodt CA, England L, Gomes DJ, et al. Characteristics associated with hospitalization among patients with COVID-19-Metropolitan Atlanta, Georgia, March-April 2020. Morb Mortal Wkly Rep. 2020;69(25):790-4.

7. Gold JA. Characteristics and clinical outcomes of adult patients hospitalized with COVID-19-Georgia, March 2020. MMWR Morb Mortal Wkly Rep. 2020:69.
8. Price-Haywood EG, Burton J, Fort D, Seoane L. Hospitalization and mortality among Black patients and White patients with Covid19. N Engl J Med. 2020;382(26):2534-43.

9. Development, O.o.R. Overview of VA research on Health Equity. 2020 cited 2020; Available from: https://www.research.va.gov/ topics/health_equity.cfm.

10. Peterson K, Anderson J, Boundy E, Ferguson L, McCleery E, Waldrip K. Mortality disparities in racial/ethnic minority groups in the Veterans Health Administration: an evidence review and map. Am J Public Health. 2018;108(3):e1-e11.

11. Selim AJ, Fincke G, Berlowitz DR, Cong Z, Miller DR, Ren XS, et al. No racial differences in mortality found among Veterans Health Administration out-patients. J Clin Epidemiol. 2004;57(5):539-42.

12. Burgess DJ, van Ryn M, Grill J, Noorbaloochi S, Griffin JM, Ricards J, et al. Presence and correlates of racial disparities in adherence to colorectal cancer screening guidelines. J Gen Intern Med. 2011;26(3):251-8.

13. Atkins D, Kilbourne A, Lipson L. Health equity research in the Veterans Health Administration: we've come far but aren't there yet. Am Publ Health Assoc. 2014.

14. Bhargava A, Kim T, Quine DB, Hauser RG. A 20-year evaluation of LOINC in the United States' largest integrated health system. Arch Pathol Lab Med. 2020;144(4):478-84.

15. Mor M. Assessing race and ethnicity. Pittsburgh: Veterans Affairs VIReC Database and Methods Cyberseminar Series; 2014. Retrieved from http://www.hsrd.research.va.gov/for_researchers/ cyber_seminars/archives/763-notes. pdf

16. Maynard C. Ascertaining veterans' vital status: VA data sources for mortality ascertainment and cause of death. Database \& Methods Cyberseminar Series. 2017.

17. Wilcox AJ. Length of intensive care unit stay computed from the VHA corporate data warehouse. NEGSUG2012. Baltimore, Maryland, 2012.

18. Scott DuVall JS. Introduction to the VA COVID-19 shared data resource and its use for research. 2020. Available from: https:// www.hsrd.research.va.gov/cyberseminars/catalog-upcomingsession.cfm?UID=3810.

19. Elixhauser A, Steiner C, Harris DR, Coffey RM. Comorbidity measures for use with administrative data. Med Care. 1998;36:8-27.

20. CDC. Evidence used to update the list of underlying medical conditions that increase a person's risk of severe illness from COVID19. 2019.

21. Wadhera RK, Wadhera P, Gaba P, Figueroa JF, Joynt Maddox KE, Yeh RW, et al. Variation in COVID-19 hospitalizations and deaths across New York City boroughs. JAMA. 2020;323:2192-5.

22. Pan D, Sze S, Minhas JS, Bangash MN, Pareek N, Divall P, et al. Ta systematic review. EClinicalMedicine. 2020;23:100404.

23. de Lusignan S, et al. Risk factors for SARS-CoV-2 among patients in the Oxford Royal College of General Practitioners Research and Surveillance Centre primary care network: a cross-sectional study. Lancet Infect Dis. 2020.

24. Dyer O. Covid-19: Black people and other minorities are hardest hit in US. Br Med J. 2020; Publishing Group.

25. Fiscella K, Franks $\mathrm{P}$, Gold MR, Clancy CM. Inequality in quality: addressing socioeconomic, racial, and ethnic disparities in health care. JAMA. 2000;283(19):2579-84.

26. Haro E, Mader M, Noël PH, Garcia H, Vogt D, Bernardy N, et al. The impact of trust, satisfaction, and perceived quality on preference for setting of future care among veterans with PTSD. Mil Med. 2019;184(11-12):e708-14.

27. Kondo K, et al., Prevalence of and interventions to reduce health disparities in vulnerable veteran populations: a map of the evidence. 2017.

28. Kondo $\mathrm{K}$, et al. Health disparities in veterans: a map of the evidence. Med Care. 2017;55(Suppl 9 Suppl 2):S9-S15. 
29. Saha S, Freeman M, Toure J, Tippens KM, Weeks C, Ibrahim S. Racial and ethnic disparities in the VA health care system: a systematic review. J Gen Intern Med. 2008;23(5):654-71.

30. Gollust SE, et al. What causes racial health care disparities? A mixed-methods study reveals variability in how health care providers perceive causal attributions. INQUIRY: J Health Care Organ Prov Financ. 2018;55:0046958018762840.

Publisher's Note Springer Nature remains neutral with regard to jurisdictional claims in published maps and institutional affiliations. 